\title{
Un âge critique. La ménopause sous le regard des médecins des XVIIIe et XIXe siècles
}

Annick Tillier

\section{OpenEdition}

1 Journals

Édition électronique

URL : https://journals.openedition.org/clio/1471

DOI : 10.4000/clio. 1471

ISSN : 1777-5299

Éditeur

Belin

Édition imprimée

Date de publication : 1 avril 2005

Pagination : 269-280

ISBN : 2-85816-781-8

ISSN : 1252-7017

Référence électronique

Annick Tillier, « Un âge critique. La ménopause sous le regard des médecins des XVIIle et XIXe siècles », Clio. Histoire, femmes et sociétés [En ligne], 21 | 2005, mis en ligne le 01 juin 2007, consulté le 22 avril 2022. URL : http://journals.openedition.org/clio/1471 ; DOI : https://doi.org/10.4000/clio.1471

Ce document a été généré automatiquement le 22 avril 2022

Tous droits réservés 


\title{
Un âge critique. La ménopause sous le regard des médecins des XVIIIe et XIXe siècles
}

\author{
Annick Tillier
}

1 Médecins et philosophes, se heurtant à l'énigme de la procréation, ont longtemps disserté sur l'anatomie et la physiologie féminines. Du début du XVI ${ }^{\mathrm{e}}$ siècle jusqu'aux années 1880 , les traités sur les «maladies des femmes » abondent. Au lendemain des Lumières, les tableaux de la nature féminine dressés par Pierre Roussel (1775) ou Pierre Moreau de la Sarthe (1803) donnent lieu à d'innombrables mais pâles copies; puis l'intérêt semble se concentrer sur la menstruation: on recense ainsi, au XIX siècle, plus de 200 thèses sur l'irruption des règles, leurs aléas, leur cessation ${ }^{1}$. Le premier livre français entièrement consacré à la ménopause est sans doute celui de Jean-Baptiste Jeannet des Longrois : Conseils aux femmes de quarante ans, publié en 1787. Il fait écho à un article du médecin anglais John Fothergill: «On the management proper at the cessation of the menses ", paru en 1776 dans le périodique de la Société médicale de Londres, qui connaît dès sa publication un grand retentissement. Traduit en français dès 1788 sous le titre Conseils aux femmes de 45 à 50 ans sur la conduite à tenir lors de la cessation des règles, réédité en 1799 et 1812, et constamment cité, il ouvre la voie à une série de publications destinées aux femmes, visant, par un ensemble de prescriptions hygiéniques, à les aider à traverser cette période longtemps qualifiée d'« âge critique ». Le vocable "ménopause » n'apparaît en effet qu'au début du XIX ${ }^{e}$ siècle. On le doit à Charles Gardanne, médecin de charité du $3^{e}$ arrondissement de Paris qui, après avoir, dans un ouvrage intitulé Avis aux femmes qui entrent dans l'âge critique (1816), employé le terme "ménespausie ", préfère adopter, dans la deuxième édition de cet ouvrage (1821), celui de ménopause ${ }^{2}$.

Une mort symbolique

2 Dans les divers d'écrits qui lui sont consacrés, la ménopause paraît à plus d'un titre justifier l'expression d'âge critique. Elle marque à la fois la mort d'un organe - l'utérus - perçu comme attribut primordial de la femme, la fin d'un processus - la menstruation 
- présenté comme régulateur de toute son économie et l'approche de la décrépitude. La ménopause est donc porteuse d'une forte charge symbolique. En perdant la faculté de procréer, la femme se voit tout d'abord privée de l'extraordinaire pouvoir qu'elle détenait dans la reproduction de l'espèce et la perpétuation des générations. En second lieu, cette perte et les phénomènes de vieillissement qui l'accompagnent peuvent être vécus selon l'expression de Moreau de la Sarthe ${ }^{3}$ comme une "mort partielle", annonciatrice de la mort naturelle. La femme, enfin, cesse d'incarner les vertus sociales attachées à la maternité et, la procréation étant l'ultime fin du mariage, l'amour même lui devient superflu :

Lorsque la mort du sexe est consommée, les femmes n'offrent plus dans leur existence aucun événement, aucunes fonctions qui exigent une direction particulière des facultés et des moyens de la vie: les agitations du cœur et de l'esprit cessent de leur être nécessaires en cessant de leur être utiles ; elles doivent se borner à arrêter le bonheur et à l'empêcher de fuir, en se livrant en paix aux affections durables et aux vertus domestiques.

3 Théodore Pétrequin va même jusqu'à décrire la ménopause comme le moment où « la femme, dépouillant peu à peu ses caractères distinctifs [sic] cesse, pour ainsi dire, d'être femme en perdant la faculté de devenir mère $»^{5}$.

Jusque tard dans le XIXe siècle, l'utérus occupe une place centrale dans les systèmes qui s'attachent à expliquer le fonctionnement de l'organisme féminin. En 1821, Charles Gardanne considère encore que c'est avec juste raison qu'«Arétée, réfléchissant sur l'énergie étonnante de la matrice, a prouvé, après Hippocrate, qu'on pourrait la regarder comme un animal vivant dans un autre animal $»^{6}$. Toutefois, cette prééminence commence à être contestée, en particulier dans la thèse soutenue en 1827 par le futur médecin Hubert Poirson. Mais pour longtemps encore, c'est plus souvent à la mort de cet organe qu'à la mise en sommeil de l'activité ovarienne qu'est attribuée la survenue de la ménopause. En 1842, Brierre de Boismont, aliéniste et auteur d'importants travaux sur la menstruation reprend à son compte, dans un ouvrage couronné par l'Académie royale de médecine, l'assertion de Cabanis selon laquelle les organes de la génération renonçant difficilement à leur activité, certaines femmes, dix ou douze ans après la cessation des règles, ressentent encore chaque mois une pléthore locale et des pressions à l'utérus. Et il se livre à de savants calculs pour démontrer combien la vie de la femme est dominée par sa physiologie :

Le total des jours employés par la nature à la menstruation est réellement effrayant ; chez une femme réglée huit jours pendant trente ans, huit années sont consacrées à cette fonction. Si vous ajoutez à ce premier fait, les grossesses, les suites de couches, les suppressions, vous aurez une idée de l'influence de l'utérus sur la vie des femmes ${ }^{\text {? }}$.

L'« enfer des femmes»

5 À lire les textes des médecins, la vie de la femme, tout entière subordonnée à la menstruation, est bornée par deux étapes essentielles, réputées tout aussi dangereuses : la puberté, qui signe l'accès à la féminité et la ménopause, qui la clôt. Parce que la régularité des règles est considérée comme une condition indispensable à l'organisme féminin, enclin à la pléthore, leur disparition introduit une révolution telle dans son économie que la santé de la femme est désormais menacée de mille périls. En référence, sans doute, à une maxime attribuée à La Rochefoucauld, certains auteurs comme Pierre Roussel assimilent la période de la ménopause à un véritable enfer8. 
6 Comme il se doit, la métaphore infernale appliquée à la vieillesse féminine comporte une dimension moralisatrice. Elle stigmatise les comportements peu conformes à l'idéal féminin qu'ont édifié les philosophes et les médecins du XVIIIe siècle. Les maladies de l'âge critique, en effet ne frappent pas indifféremment toutes les femmes. Cet "enfer " n'est qu'un juste châtiment venant sanctionner celles qui ont refusé d'assumer leur féminité ou qui, par leur conduite excessive, ont enfreint les convenances. Les femmes qui ont usé convenablement de la vie, qui ont été mères, sont en effet censées éprouver moins de difficultés à traverser cet "âge dangereux" que celles dont la vie a été perturbée par les chagrins ou trop agitée et celles qui, refusant d'endosser les rôles sociaux féminins, sont demeurées dans le célibat. La chasteté est considérée comme très néfaste à la santé des femmes. Moreau de la Sarthe décrit les célibataires comme de "malheureuses victimes d'une douloureuse virginité [...] qui ont été privées des avantages qui devaient résulter de la jouissance pleine et entière de leurs facultés 9 . Pour Julien Virey, la virginité est un "état de violence contre les impulsions de la nature » et les religieuses cloîtrées, célibataires par excellence, souffrent très souvent de troubles tels que la chlorose, l'aménorrhée, les accès hystériques ou les spasmes nerveux. La sobriété est exaltée et la vie des campagnardes, qui partagent harmonieusement leur temps entre le travail et le repos, se contentent d'une nourriture simple et peu abondante, et mènent une vie active est érigée en modèle. En revanche, les femmes qui ont abusé de la bonne chère et de l'alcool et qui, de ce fait ont eu des menstrues très abondantes, "éprouvent à l'époque de leur cessation des affections singulièrement variées, ou les ressentent à un plus haut niveau d'exaspération ${ }^{10}$. L'oisiveté des femmes aisées est stigmatisée ; leur inactivité favorise pléthore et embonpoint, tandis que les fêtes, les spectacles, les divertissements auxquels elles consacrent leurs nuits entretiennent un état d'agitation perpétuel nuisible à leur organisme. En contrepoint et bien que, selon Roussel, "les grâces s'accommodent peu de la sueur et du hâle », la conduite sobre et vertueuse des femmes des classes laborieuses est glorifiée.

7 La dégénérescence des organes génitaux est supposée entraîner chez la femme une véritable révolution. Telle une fleur délicate qui perd soudain son éclat et sa vitalité, « l'automne » de sa vie est marquée par une inexorable métamorphose :

Le quatrième âge s'annonce dans la destruction graduelle de tout ce qui a le plus brillé dans les âges précédents. Le tempérament change, la constitution se détériore, l'abdomen se grossit, perd son poli, les traces de la maternité forment des stigmates qui altèrent plus ou moins la peau qui les recouvre, le cou s'amaigrit, les mamelles devenues inutiles sont longues et pendantes, les altérations des formes se multiplient, les clavicules forment une saillie désagréable, la taille perd de son élégance, et les mouvements commencent à devenir plus difficiles $»^{11}$.

8 Ces modifications sont d'autant plus brutales que si la jeunesse de la femme est, selon Roussel, plus brillante que celle de l'homme, sa vieillesse est également plus hâtive. Dépouillée des caractéristiques de son sexe, la femme qui atteint l'âge de la ménopause perd à jamais sa séduction. Non seulement ses traits, sa physionomie subissent des modifications considérables, mais ses idées, ses goûts, sa sensibilité tendent désormais à se rapprocher de ceux de l'homme. Moreau de la Sarthe et, après lui Édouard Aubert, croient pouvoir les comparer à des eunuques :

$\mathrm{Si}$, chez les Scytes, dont les historiens ont vanté la sagesse, les hommes qui perdaient leurs facultés viriles étaient obligés de prendre les vêtements et les habitudes des femmes, pourquoi celles-ci, lorsque leur faculté d'engendrer est abolie, ne pourraient-elles pas appartenir à la classe des hommes sous plusieurs 
rapports, et jouir, à ce titre des mêmes privilèges, ou se livrer à des professions libérales, à des travaux littéraires qui souvent leur offriraient des moyens d'existence et de consolation ? $^{12}$ le temps qu'il leur reste à vivre à des activités - écriture, travaux intellectuels - qui leur étaient jusque là sinon formellement interdites du moins fortement déconseillées. Roussel, en effet, estimait que l'étude, par la contention de l'esprit qu'elle impose, était susceptible de détériorer le tempérament féminin. Cabanis considérait les femmes «savantes" comme des «êtres incertains qui ne sont, à proprement parler d'aucun sexe $»^{13}$.

Les maladies de l'âge critique maladies sont susceptibles d'assaillir les femmes lorsque la menstruation s'achève. Bouffées de chaleur, vapeurs, insomnies, rêves fatiguants, difficultés respiratoires, spasmes, troubles digestifs, hémorroïdes, infections de la matrice, voire cancers, polypes, hémorragies en tout genre, angines, ophtalmies, affections cutanées et rhumatismales, maladies nerveuses..., l'éventail des maladies évoquées est infini. Leur origine est, bien entendu, souvent attribuée à l'utérus, qui selon Moreau de la Sarthe dans un «dernier effort pour conserver son empire et sa prédominance d'action, bouleverse tout le système vivant $~^{14}$. Mais d'autres médecins les mettent au compte du racornissement des ovaires ou, tel Fothergill, du rétrécissement des vaisseaux. La pléthore et les phénomènes congestifs qui en résultent sont d'autant plus dangereux que la nature de la femme - humide et spongieuse selon la tradition hippocratique favorise le croupissement des humeurs.

11 Ces publications contribuent à alimenter la peur, si ce n'est la terreur, qui paraît s'emparer des femmes qui approchent de la cinquantaine. Certains médecins s'efforcent de les rassurer. Fothergill, imité par beaucoup d'autres, considère que les craintes des femmes reposent sur de fausses conceptions du sang menstruel, répandues d'âge en âge depuis l'Antiquité, qui lui attribuent un caractère morbifique :

Nous savons actuellement que le flux menstruel n'est pas ce que la plupart des personnes du sexe ont trop longtemps et trop généralement cru, l'évacuation d'une humeur peccante et morbifique, quelquefois âcre et maligne, dont la rétention ne manque jamais d'être nuisible à la constitution par ses qualités délétères [...]. Il est actuellement reconnu, et le sexe ne saurait être trop bien instruit de cette vérité, que le flux menstruel ne possède aucune pareille malignité, qu'il n'est qu'une abondance de ce sang vital et pur qui anime tout le système d'une personne bien portante, et que sa suppression en général n'est nullement accompagnée d'effets qui soient plus difficiles à dissiper que toute autre affection à laquelle elles sont sujettes.

Et il cite des exemples de patientes qui, loin de voir leur santé se détériorer, retrouvent une seconde jeunesse. Ce rajeunissement - bien qu'éphémère - explique aux yeux de Menville l'expression "âge de retour ", souvent utilisée pour évoquer la période de la ménopause. D'autres auteurs, en revanche, associent plutôt cette expression au retour sur soi que doit opérer la femme qui, abordant l'âge du déclin, est contrainte d'adopter d'autres habitudes de vie. Cette vision plutôt rassurante du vieillissement, reprise par Poirson, Saucerotte, Brierre de Boismont, Ernest Barié... est confortée par les travaux des statisticiens de la population qui, à la suite de Benoiston de Châteauneuf, remarquent que le taux de mortalité des femmes n'augmente pas de manière significative entre 40 et 50 ans. Benoiston de Châteauneuf, démontre même, d'après des 
statistiques recueillies dans diverses contrées d'Europe, que la mortalité des hommes du même âge est de trois à quatre fois supérieure, ce qui l'amène à conclure qu'« il serait exact de dire que cette époque est réellement l'époque critique des hommes, et non celle des femmes $»^{15}$. D'autres observateurs, ayant travaillé sur des corpus plus restreints et sans doute plus fiables, avaient abouti aux mêmes conclusions, notamment Claude Lachaise, auteur d'une Topographie médicale de Paris (1822).

Une des questions que se posent les médecins est de savoir si la crise morale suscitée par la ménopause peut être à l'origine de maladies mentales. Dans sa célèbre étude sur le cas d'Henriette Cornier, meurtrière d'une petite fille, l'aliéniste Marc avait insisté sur le fait que cette accusée était à l'époque de ses règles au moment du crime et mis l'accent sur l'influence exercée par la menstruation dans la production de la monomanie. Dans les premières années décennies du XIX ${ }^{e}$ siècle Pinel, Esquirol, Voisin, Georget, ... les plus grands aliénistes s'interrogent sur l'influence que peut exercer cette période de la vie féminine sur le déclenchement des névroses, en particulier de l'hystérie ou de l'épilepsie. Mais, en dehors des cas où les femmes examinées présentaient déjà des prédispositions, ni les observations cliniques ni les statistiques n'en fournissent la démonstration. Tout juste Esquirol peut-il déterminer que la ménopause favorise le développement de la mélancolie ${ }^{16}$. Aussi leurs successeurs préfèrent-ils s'en tenir à la qualification assez vague de nervosisme ou à celle, non moins imprécise de "névropathie protéiforme ", attribuée à Laurent Cerise et qu'il définit ainsi :

Douleurs de tête, vertiges, hallucinations, étouffement, météorisme, vomissements, palpitations, abattement, agitation, graves hémorragies, brusques suppressions, somnolence invincible, insomnie opiniâtre, rêves, cauchemars, inappétence, dégoût, chaleur, frisson, spasmes, convulsions, etc., tout cela alterne, se succède, se mêle, se combine pour torturer l'infortunée victime des maux de nerfs ${ }^{17}$.

14 Presque tous les médecins s'accordent à déceler, chez la femme qui aborde la ménopause, un rebond de sexualité. Chambon de Montaux l'attribue à des phénomènes congestifs :

une sorte d'embarras dans la circulation, cause très souvent un trouble qui s'annonce par des symptômes dont on n'a pas encore développé la théorie : c'est le désir violent, chez quelques personnes, des plaisirs de l'amour. Désir que la réflexion repousse quelquefois longtemps, mais dont l'empire est souvent au-dessus de la raison, dans les sujets d'une constitution vigoureuse, et surtout chez les femmes qui ont été modérées dans leur jouissance. Cette maladie annonce une sorte de congestion dans les organes de la génération, elle y cause une irritation, d'abord légère et difficile à connaître, qui ramène dans le cœur cette sensibilité de laquelle on ne se défie point encore; qu'à cet âge peut-être une femme vertueuse prend souvent pour un sentiment d'amitié plus vif et plus empressé18

15 Condamnée à réprimer des désirs qu'elle n'ose plus satisfaire, la malheureuse femme en proie de ces accès vénériens est vouée à l'ennui, à la solitude, à la douleur. Vaines chimères, regrets, tourments... toutes sortes de sentiments et d'émotions semblent submerger les femmes qui voient disparaitre leurs attraits. Elles deviennent, écrit Charles Menville, "moroses, taciturnes, inquiètes, irritables et même méchantes $»^{19}$. Élevées pour séduire, rien ne prépare les femmes qui se voient privées de leurs agréments, à surmonter sereinement cette douloureuse épreuve, déplorait déjà, au siècle précédent la marquise de Lambert ${ }^{20}$.

16 Certains médecins se complaisent à décrire l'affolement qui s'empare des femmes à l'approche de la cinquantaine et les stratagèmes qu'elles emploient pour dissimuler les 
ravages du temps. Astruc fait état des difficultés qu'il éprouve avec des patientes qui, refusant la disparition de leur règles, lui demandent de les faire " revenir » :

Par malheur il y a des femmes qui ne se croient jamais assez âgées pour perdre leurs règles, et qui en se trompant, trompent leurs Médecins; il y en a d'autres qui peuvent bien avoir quelque soupçon de la vérité, mais qui n'en ont que plus de soin de la cacher aux autres; et dans cet entêtement elles cherchent encore avec plus d'ardeur à faire illusion à leurs Médecins. Elles sont ordinairement les victimes de leur légèreté. ou de leur caprice, car, en s'attachant à rappeler des règles qui devraient être cessées, et à forcer la nature, elles s'attirent souvent des pertes de sang, des fleurs blanches habituelles, ou ce qui est encore plus dangereux, des inflammations, des ulcères, des cancers dans la matrice ${ }^{21}$.

17 De nombreux médecins condamnent les fards et autres artifices utilisés pour simuler l'éclat et la fermeté de la jeunesse: «Le ridicule est à son comble, écrit Gardanne, lorsqu'on voit des femmes de cinquante ans se vêtir comme de jeunes personnes, les imiter dans toutes leurs manières, aller les bras et la poitrine découverts, faire supporter avec prétention, par des mécaniques, ce que la nature soutient elle-même chez la jeune personne, mécaniques qui exercent des compressions dont les plus légères suffisent pour produire, à cet âge, des engorgemens squirrheux, des cancers aux seins, à l'estomac, etc. $\aleph^{22}$. Ce discours dépréciatif n'est pas exclusivement masculin. Virginie Messager, maîtresse sage-femme, l'une des rares femmes à avoir rédigé un Guide pratique de l'âge critique, ${ }^{23}$ dépeint les troubles de l'humeur que suscite cette perte du pouvoir de séduction :

Toutes, celles surtout qui attachaient beaucoup d'importance à leur beauté et aux jouissances de toute nature qu'elle leur procurait, reconnaissant que leurs charmes pourraient bien s'évanouir, deviennent inquiètes, irritables, jalouses : il semble qu'elles cherchent à se venger sur tous ceux qui les environnent des menaces du temps et des chagrins qu'il va leur causer $»^{24}$.

18 Marie de Thilo, médecin suisse, compte aussi au nombre des troubles consécutifs à la crise morale que traversent les femmes à l'âge critique mélancolie et idées fixes : «On en voit [des femmes] qui sont obsédées par des imaginations plus ou moins absurdes [...]. D'autres sont en proie à la monomanie mystique : on voit même quelquefois éclater des crises véritables de folie ou mélancolie $»^{25}$.

Conseils et remèdes

19 Les prescriptions hygiéniques et remèdes proposés pour surmonter l'âge critique sont marqués par une conception encore très hippocratique de la médecine, qui accorde une grande importance aux humeurs, aux saisons et aux climats. Être de nature, la femme est réputée particulièrement sensible aux effets de la chaleur ou du froid, de l'humidité et des vents. Les conseils d'hygiène contenus dans les ouvrages destinés aux femmes sont généralement énoncés dans l'ordre de la classification de Hallé26 et se réfèrent successivement à l'action des «choses environnantes» (air, climat, ...), des «choses appliquées à la surface du corps » (vêtements, bains, cosmétiques, ...), des substances ingérées ou excrétées, de l'exercice et du repos et, enfin, à l'influence des perceptions, sensations, affections de l'âme.

20 La femme ménopausée est invitée à éviter les atmosphères échauffées que constituent par exemple les salons très fréquentés ou les salles de spectacle. Si elle à la possibilité de choisir son habitation, elle doit fuir les lieux humides et exposés au vent, les eaux stagnantes, les appartements trop proches du sol. Elle doit veiller à se protéger du froid, tout en ne se couvrant que modérément. La nourriture exige une égale attention. Les aliments « échauffants ", en particulier les viandes rouges, ainsi que l'alcool sont à 
proscrire, au profit des « délayants » que sont végétaux et eaux minérales, qui aident à fluidifier un sang qui s'est épaissi, et des laitages. Celle qui souffre de bouffées de chaleur, de vapeurs, d'insomnies est invitée à se dispenser du repas du soir.

21 Sa propension naturelle à la pléthore étant aggravée par la fin de la menstruation, la femme doit lutter contre la stase des humeurs en exerçant une activité physique régulière mais modérée, comme la promenade - matinale de préférence, ce qui lui évite de s'attarder au lit - ou les travaux ménagers: "Aux unes il faut conseiller de s'adonner au jardinage, de bêcher la terre, de sarcler le jardin et d'arroser les fleurs ; aux autres des exercices plus actifs sont indispensables: on leur recommandera de frotter les appartements, de fendre ou de scier du bois $»^{27}$. Les activités domestiques présentent l'avantage de joindre au bien-être physique procuré la satisfaction du devoir accompli. Les bains, ni trop chauds, ni trop froids, peuvent aider à lutter contre la pléthore, de même que les saignées, bien que certains médecins les estiment dangereuses. En 1852, cependant, c'est encore par la lancette qu'est traitée George Sand qui souffre des troubles de la ménopause. «Le sang s'est porté à la poitrine et j'ai tous les accidents de la phtisie, confie-t-elle à sa sœur le 6 février. Mon médecin assure que c'est l'effet de l'âge critique et qu'il me guérira ${ }^{28}$. Saignée le lendemain, elle se voit également administrer d'autres remèdes décongestionnants qui lui apportent un répit momentané. Aussi écrit-elle quelques jours plus tard : « On m’a saignée, synapisée [sic], vomitisée, morphinisée, et après toutes ces secousses qui m'ont mise sur les dents quatre ou cinq fois, on m'a enfin débarrassée de l'étouffement qui me paralysait ${ }^{29}$.

Lorsque vient l'âge de la ménopause, les femmes, devenues plus sensibles aux effets des passions, sont invitées à se retirer du monde, à renoncer à leurs habitudes frivoles, au profit de distractions apaisantes comme la lecture - mais les ouvrages licencieux sont proscrits - la conversation, les réunions d'amis, les voyages, les séjours à la campagne et à se garder de toute activité susceptible d'échauffer leur organisme. Pour cela, elles doivent fuir les sources d'excitation que constituent fêtes et bals et veiller à modérer leurs émotions et leurs sentiments. Toutes ces agitations du cœur « ne conviennent pas aux femmes qui ont dépassé l'âge de la quarantaine » écrit par exemple Moreau de la Sarthe. Elles doivent, surtout, se résoudre à renoncer au plaisir vénérien : «L'amour, ce beau soleil de la vie, doit s'éteindre pour toujours dans le cœur de la femme qui n'existe que pour la reproduction $»^{30}$. Plus que jamais, la femme est assignée à l'espace privé et à la douce chaleur du foyer. Mais elle peut trouver, si elle a su cultiver suffisamment de vertus pour se rendre aimable, des consolations dans l'amitié et surtout dans l'amour de ses enfants :

La nature sage et prévoyante offre à la mère, qui a été assez vertueuse pour être docile à sa voix, des jouissances qui ne finissent qu'avec la vie : la beauté, les grâces ne sont point perdues pour elles, puisqu'elle les a transmises à ses enfants ; l'intérêt qu'inspirent leurs ingénues candeurs et leur heureuse physionomie, sont des hommages secrets qu'on rend à elles-mêmes, et qui lui rappellent toujours, avec l'émotion du plaisir, le temps où elle en était le véritable objet ${ }^{31}$. 


\section{BIBLIOGRAPHIE}

ASTRUC Jean, 1761-1765, Traité des maladies des femmes, Paris, P. Guillaume Cavelier, 5 vol.

AUBERT Édouard, 1841, Hygiène des femmes nerveuses, Paris, Germer Baillière.

BARIÉ Ernest, 1877, Étude sur la ménopause, Paris, impr. A. Parent.

BENOISTON DE CHATEAUNEUF Louis-François, 1822, Mémoire sur la mortalité des femmes de l'âge de quarante à cinquante ans, lu à l'Académie des sciences dans sa séance du 13 mai 1818, Paris, chez Martinet et chez l'auteur.

BRIERRE DE BOIMONT Alexandre, 1842, De la menstruation considérée dans ses rapports physiologiques et pathologiques, Paris, G. Baillière.

CABANIS Pierre-Jean-Georges, 1844, Rapports du physique et du moral de l'homme et lettres sur les causes premières, $8^{\mathrm{e}}$ éd., Paris, J.-B. Baillière [1 $1^{\text {ère }}$ éd. 1802].

CERISE Laurent, 1845, « Du rôle des émotions dans la vie de la femme », Introduction à Pierre Roussel, Système physique et moral de la femme, Paris, Fortin, Masson et $\mathrm{C}^{\mathrm{ie}}$, Charpentier.

CHAMBON DE MONTAUX Nicolas, 1784, Des Maladies des femmes, Paris, rue et Hôtel Serpente, 2 vol.

DEBAY Auguste, 1862, Histoire naturelle de l'homme et de la femme, Paris, E. Dentu.

ESQUIROL Jean-Étienne-Dominique, 1838, Des Maladies mentales considérées sous les rapports médical, hygiénique et médico-légal, Paris, J. B. Baillière.

FOTHERGILL John, 1776, « On the management proper at the cessation of the menses », Medical observations and inquiries, V, p. 160-186.

GARDANNE Charles, 1821, De la ménopause ou de l'âge critique des femmes, $2^{\mathrm{de}}$ éd. Paris, MéquignonMarvis,

GUYOT Gilbert, 1817, Essai sur la femme considérée dans les différentes périodes de sa vie. [S.l. : s. n.]

LA ROCHEFOUCAULD François, duc de. Maximes, suivies de réflexions diverses [...] Texte établi par Jacques Truchet, $3^{e}$ éd., Paris, Bordas, 1985. (Classiques Garnier).

LAMBERT Anne-Thérèse Marguenat de Courcelles, marquise de Lambert, Traité de la vieillesse, in CEuvres, 1990, Paris, H. Champion [1 ${ }^{\text {ère }}$ éd. 1747].

MENVILLE Charles, 1840, De l'âge critique chez les femmes, des maladies qui peuvent survenir à cette époque de la vie et des moyens de les combattre et de les prévenir, Paris, Germer Baillière

MESSAGER Virginie, 1859, Guide pratique de l'âge critique ou conseils aux femmes sur les maladies qui peuvent les attaquer à cette époque de leur vie, et sur les moyens de combattre ces maladies, mais surtout de les prévenir [...], Paris, chez l'auteur.

MOREAU DE LA SARTHE Pierre, 1803, Histoire naturelle de la femme, suivie d'un traité d'hygiène appliquée à son régime physique et moral aux différentes époques de sa vie, Paris, L. Duprat, Letellier et Comp ${ }^{\text {ie. }}$

PÉTREQUIN Théodore, 1836, Recherches sur la menstruation, Paris, impr. de Didot le jeune.

PINEL Philippe, 1803, Nosographie philosophique ou la méthode de l'analyse appliquée à la médecine. $5^{\mathrm{e}}$

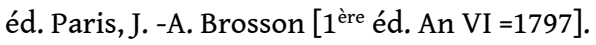


POIRSON Hubert, 1827, Dissertation sur la ménespausie. Strasbourg, impr. de F. G. Levrault.

ROUSSEL Pierre, 1845, Système physique et moral de la femme. Nouv. éd. Paris, Fortin, Masson et Cier, Charpentier [1 ${ }^{\text {ère }}$ ed. 1775].

SAND George, 1973, Correspondance. Textes réunis, classés et annotés par Georges Lubin. Tome 10 (janvier 1851 - mars 1852). Paris, Garnier frères, 1973.

SAUCEROTTE Constant, 1828, Nouveaux conseils aux femmes sur l'âge prétendu critique ou conduite à tenir lors de la cessation des règles. Paris, Madame Auger-Méquignon et Delaunay.

THILO Marie de, 1891, L'hygiène de la femme, Paris, Fishbacher.

VIREY Julien-Joseph, 1823, De la femme sous ses rapports physiologiques, moral et littéraire, Paris, Crochard.

Études récentes citées

ARNAUD Robert, 1996, La ménopause à travers l'histoire, Rueil-Malmaison, Laboratoires Ciba-Geigy, 1996.

TILLIER Annick, 2004, « De quelques images de la femme dans les écrits médicaux des XVIII et $\mathrm{XIX}^{\mathrm{e}}$ siècles ", Revue de la Bibliothèque nationale de France, 17, p. 68-72.

\section{NOTES}

1. Tillier $2004: 68-72$.

2. Arnaud 1996.

3. Moreau de la Sarthe 1803 : II, 372.

4. Guyot $1817: 19$.

5. Pétrequin $1836: 11$.

6. Gardanne $1821: 17-18$.

7. Brierre de Boimont 1842 : IX-X.

8. «L'enfer des femmes c'est la vieillesse ».

9. Moreau de la Sarthe 1803 : I, 407-408.

10. Pinel $1813: 645$.

11. Guyot $1817: 17$.

12. Moreau de la Sarthe 1803 : II, 382.

13. Cabanis $1844: 307$ [ 1 ère éd. 1802].

14. Moreau de la Sarthe 1803 I : 408.

15. Benoiston de Chateauneuf $1822: 13$.

16. Esquirol $1838: \mathrm{I}, 434-435$

17. Cerise 1845 : XLIX.

18. Chambon de Montaux 1784 : II, 266.

19. Menville $1840: 160$.

20. Lambert $1990: 181$ [1 $1^{\text {ère }}$ éd. 1747].

21. Astruc 1761 : I, 156.

22. Gardanne 1821: 124.

23. En France il faut attendre 1875 pour qu'une femme - Madeleine Brès - soit admise à se présenter au doctorat de médecine.

24. Messager $1859: 50$.

25. Thilo $1891: 88$. 
26. Circumfusa, applicata, ingesta, excreta, gesta, percepta

27. Brierre de Boimont $1842: 250$.

28. Sand 1973, Lettre à Caroline Cazamajou, 6 février 1852.

29. Sand 1973, Lettre à Pierre-Jules Hetzel, 18 février 1852.

30. Debay $1862: 445$.

31. Guoyot $1817: 20$.

\section{RÉSUMÉS}

Si l'on possède peu de témoignages sur la manière dont les femmes du XIXe siècle ont vécu le vieillissement, les discours des médecins sur cette question abondent. La ménopause est décrite par eux comme une période particulièrement dangereuse qui, à l'instar de la puberté, bouleverse toute l'économie de la femme. Au nombre des maladies qui sont susceptibles de l'assaillir lorsque s'interrompt le mécanisme régulateur que représentait la menstruation s'ajoute la blessure narcissique que provoque la perte de sa féminité et l'entrée dans l'âge de décrépitude. Plus encore, la femme perdant avec la faculté d'engendrer sa vocation sociale (la maternité), cette période, souvent qualifiée d'âge critique ou d'âge dangereux, s'annonce comme une véritable mort sociale. Privée de sa capacité de séduction, fragilisée par la révolution physiologique qui s'opère en elle, la femme, encouragée à se retirer d'un monde où elle ne peut plus briller, est plus que jamais assignée à la sphère privée.

There are but few testimonies concerning the way 19th-century women experienced aging. What we do have, however, are doctors' discourses on the subject. They describe menopause as a particularly dangerous period, which, just like puberty, upset women's system. Among the different diseases likely to befall women when the regulating mechanism of menstruation stopped, was a narcissistic wound due to the loss of their femininity announcing the beginning of decrepitude. Moreover, when they stopped being of childbearing age, women lost their main social function. Often called "the critical age" or "the dangerous age", this period heralds the doom of their social existence. Deprived of their ability to seduce, weakened by their physiological changes, women were encouraged to retire from a world where they could no longer shine, and were condemned, more than ever, to the private sphere.

INDEX

Mots-clés : femmes, maladies, médecins et femmes, soins médicaux, vieillissement, ménopause

\section{AUTEUR}

\section{ANNICK TILLIER}

Annick TILLIER est conservateur en chef à la Bibliothèque nationale de France, département de recherche bibliographique. Ses recherches portent sur l'histoire de la criminalité et sur l'histoire sociale de la médecine au XIXe siècle. Elle a publié : Des criminelles au village : femmes infanticides en 
Bretagne, 1825-1865, Presses universitaires de Rennes, 2001 ; «L'infanticide devant les cours d'assises en Bretagne (1825-1865) », Histoire de la justice, 1994, 7 ; « Infanticide : le procès des mères criminelles », L'Histoire, $n^{\circ} 282$, déc. 2003 ; « De quelques images de la femme dans les écrits médicaux des XVIIIe et XIXe siècles ", Revue de la Bibliothèque nationale de France, 17, 2004. 